\title{
Cambios en la dinámica de deforestación de la subcuenca de un río en México: la imposibilidad de recuperación de los hábitats originales después del cese de la deforestación ${ }^{1}$
}

\author{
Ofelia Sotelo-Caro², Josué Chichia-González ${ }^{3}$, Valentino Sorani ${ }^{4}$ \\ y Alejandro Flores-Palacios ${ }^{5}$
}

\begin{abstract}
RESUMEN
En la zona Neotropical de la sub-cuenca del río Apatlaco (centro de México), la alta deforestación puede ser atribuida al desarrollo urbano, ya que esta zona concentra la mayor densidad de población del Estado de Morelos. El objetivo de este estudio es analizar los cambios en el uso del suelo y de la vegetación en esta subcuenca entre 2002 y 2012, utilizando Sistemas de Información Geográfica. Los resultados muestran que, durante el período de análisis, los principales cambios ocurrieron en los pastizales y bosques de coníferas. Sin embargo, el bosque tropical caducifolio (BTC) sufrió una tasa anual de deforestación $(0,13 \%)$ mucho más baja que la estimada previamente $(1,4 \%)$, pero no ocurrió una recuperación. Es posible que la deforestación del BTC se haya detenido, debido a que las áreas con remanentes de bosque, son inadecuados para la agricultura u otras actividades. Es una prioridad proteger las áreas de vegetación secundaria, pues sirven como un amortiguador para las áreas fragmentadas, lo que permitiría su recuperación y contribuir a su interconectividad.
\end{abstract}

Palabras clave: Cambio de cobertura del suelo, río Apatlaco, Sistemas de Información Geográfica.

\begin{abstract}
In the Neotropical area of the Río Apatlaco sub-basin (central Mexico), high deforestation can be attributed to urban development, as this area concentrates the highest human population density in Morelos state. The objective of this study was to analyze changes in land use and vegetation in this sub-basin from 2002 to 2012 using geographic information systems. The results show that, over the period of analysis, the main changes occurred in the grassland and coniferous forest. However, in the tropical dry forest (TDF) the annual rate of deforestation $(0.13 \%)$ was much lower than previously estimated $(1.4 \%)$, but no recovery took place. It is possible that deforestation of the TDF was halted because the remaining areas with forest remnants were unsuitable for agricultural activities and/or abandonment of such activities has taken place. It is therefore a priority to protect these areas of secondary vegetation that serve as a buffer for conserved areas, allowing their recovery and contributing to their interconnectivity.
\end{abstract}

Key words: Land cover change, Rio Apatlaco, Geographic Information Systems.

1 Artículo recibido el 8 de junio de 2014, aceptado el 5 de marzo de 2015 y corregido el 7 de mayo de 2015.

2 Centro de Investigación en Biodiversidad y Conservación $(\mathrm{Cl} \beta \gamma \mathrm{C})$, Universidad Autónoma del Estado de Morelos (México).

E-mail: bióloga_ofeliasc@yahoo.com.mx

3 Dirección General de Ordenamiento Territorial. Secretaría Desarrollo Sustentable. Gobierno del Estado de Morelos (México). E-mail: josue.chichia@gmail.com
4 Centro de Investigación en Biodiversidad y Conservación $(\mathrm{C} / \beta \gamma \mathrm{C})$, Universidad Autónoma del Estado de Morelos (México). E-mail: vsorani@yahoo.com.mx

5 Centro de Investigación en Biodiversidad y Conservación $(\mathrm{Cl} \beta \gamma \mathrm{C})$, Universidad Autónoma del Estado de Morelos (México).

E-mail: alejandro.florez@maem.mx 
Estimar y monitorear la magnitud, dinámicas y causalidades de la deforestación son metas primarias para la conservación de los ecosistemas terrestres (Goetz et al., 2009). La deforestación es un fenómeno dinámico, dependiendo del periodo analizado ya que puede acelerarse, detenerse o revertirse (Ernst et al., 2013) y su monitoreo sirve para medir el grado de amenaza que enfrenta un ecosistema terrestre (Miles et al., 2006). La identificación de la tasa de cambio de uso del suelo, permite medir las condiciones en las que se encuentran los recursos naturales, al igual que dar cuenta de las tendencias de degradación y recuperación de los mismos. Las tasas de deforestación han sido empleadas para estimar la extinción aunque han dado resultados poco certeros (He \& Hubbell, 2011).

Durante el periodo comprendido entre 1990 y 2000, los bosques tropicales en México presentaron una pérdida de 5,5 millones de hectáreas $(1,1 \%$ anual) por año (FAO, 2006). De estos, el bosque tropical caducifolio (BTC) es uno de los más importantes del país, pues es el tercero más extenso (Flores y Gerez, 1994; Palacios-Prieto et al., 2000), albergando aproximadamente al 33\% (824 especies) de los vertebrados terrestres y a 6.000 especies de plantas vasculares (Ceballos \& García, 1995; Rzedowski, 1991a), de las cuales 40\% son endémicas (Rzedowski, 1991b).

En México, Flores y Gerez (1994) estimaron que la cobertura de BTC en 1981, era del $12,36 \%$ y solamente el $8,92 \%$ no presentaba perturbación. Para 1990, la cobertura nacional de BTC conservado, era de 6,98 \% y para 1992 el área deforestada anualmente se estimaba en 163.000 hectáreas (Rincón \& Huante, 1993). En el estado de Morelos, el BTC es el tipo de vegetación más extenso (ContrerasMacBeath et al., 2004), sin embargo, para el periodo comprendido entre 1973 y 1989 se calculó una pérdida del $1.4 \%$ anual (Trejo \& Dirzo, 2000). Trejo \& Dirzo (2000), sugieren que dicha tasa es una de las más altas entre los ecosistemas tropicales de México

El presente estudio tiene como objetivo medir la tasa de cambio de uso del suelo durante el periodo comprendido entre 2002 y 2012, en la subcuenca del río Apatlaco (centro de México). Esta zona se caracteriza por presentar un continuo de bosques templados a cálidos: de los primeros se desconoce la tasa de deforestación, pero están dentro de un área natural protegida, mientras que del BTC se tiene una estimación previa según la cual llegó a comprender más del 70\% de la cobertura del estado de Morelos (Trejo \& Dirzo, 2000). En consecuencia, la hipótesis general, es que la tasa de deforestación del BTC de la zona se mantuvo en 1,4\% anual (Trejo \& Dirzo 2000), o se incrementó, pues no hubo medidas de conservación, pero sí alta presión para la ampliación de la zona urbana; mientras que los bosques templados debiesen tener tasas de deforestación cercanas a cero o incluso de recuperación.

\section{Método}

La subcuenca hidrológica del río Apatlaco se localiza en el centro de México, entre los estados de Morelos, el estado de México y el Distrito Federal. Tiene un área de 85,312 ha, de la cual el $90 \%$ se localiza en el primero, representando el $13 \%$ del territorio de este (SAGARPA, 2006). La parte alta de la subcuenca alcanza los 2.550 m.s.n.m. y se caracteriza por presentar un clima semifrío, con temperatura media mensual de $16^{\circ} \mathrm{C}$, precipitación constante en el verano $(1.500 \mathrm{~mm}$ de promedio anual), mientras que en la parte baja (<890 m s.n.m.) de la subcuenca, el clima es semicálido (temperatura media mensual de $23^{\circ} \mathrm{C}$ ) y una precipitación marcadamente menor (850 mm de promedio anual).

Para delimitar el área de estudio se utilizó la información cartográfica generada por el Programa Nacional de Microcuencas (SAGARPA, 2006). Para el mapa de 2012 y la distribución potencial de BTC, se utilizaron imágenes SPOT5, correspondientes al año 2012 (tamaño de píxel de 10 x 10 m). Con estas imágenes, se generó un mosaico en el software ERDAS versión 9.0 (Fueyo, 2008), para destacar la vegetación natural y su estado de estrés. El estado de estrés se obtuvo, con las bandas $4(1,58-1,75 \mu \mathrm{m}), 3$ $(0,78$ a $0,89 \mu \mathrm{m})$ y $2(0,61$ a $0,68 \mu \mathrm{m})$ para generar el compuesto de falso color (Mas et al., 1996). Mediante el modelo digital del terreno del Instituto Nacional de Estadística, Geografía e Informática (INEGI) se ortorrectificó el mosaico, asignándole una proyección cartográfica con la escala UTM (Universal 
Transversa de Mercator: Zona 14, Datum WGS 84). Para la elaboración del mapa del año 2012, se empleó el programa ArcGis 10.1 para la fotointerpretación de las ortofotos en una escala de 1:10.000. En todos los análisis se usó la misma clasificación de los tipos de vegetación.

Para la interpretación de la distribución del BTC en las imágenes del 2012, el BTC se dividió en tres categorías: la primera categoría fue el BTC primario, que corresponde a áreas con una cobertura vegetal densa; la segunda categoría fue el BTC perturbado (BTCp), que incluye las zonas donde la cobertura arbórea no era densa en las imágenes satelitales; y la tercera categoría fue la de vegetación secundaria derivada del BTC, siendo reconocible en las imágenes satelitales porque estaban en zonas donde antes había BTC y que en las más recientes tenían escasos elementos arbóreos. Además, se hicieron recorridos de campo para verificar el tipo de vegetación con base en especies indicadoras de perturbación/conservación y la estructura fisonómica del bosque.

Para el mapa correspondiente al 2002, se interpretaron ortofotos, utilizando la misma leyenda para el año 2012. Los mapas temáticos fueron presentados en una escala de 1 : 10.000 y se calcularon las tasas de cambio para cada tipo de vegetación siguiendo el modelo exponencial (Trejo \& Dirzo, 2000). La distribución potencial de la BTC en la subcuenca se estimó con un modelo de aptitud, este modelo ponderó las zonas que tenían características para el desarrollo de BTC, usando los atributos cartográficos de edafología, litología, precipitación y el modelo digital de elevación (SEMARNAT, 2012).

La evaluación de la clasificación fue sujeta a un análisis de confiabilidad (Johnson et al., 2002), para estimar su precisión. Para análisis se usó una matriz de confusión en la que se comparó la asignación a un tipo de vegetación de 129 puntos de reconocimiento de campo, con la asignación de estos mismo puntos derivada de la interpretación de las ortofotos del año 2012. Una vez realizada la comparación se calculó la exactitud de la fotointerpretación (Anderson et al., 1976) arrojando los siguientes resultados: la exactitud de la fotointerpretación fue de $90 \%$ en forma general, mientras que su fiabilidad fue de $90 \%$ y su precisión de $91 \%$, siendo esta última más baja para el bosque de pino perturbado y el bosque de encino perturbado.

Con el fin de determinar las causas del cambio en el uso del suelo en las áreas con BTC y conocer sobre la percepción social de estos cambios, se realizaron 25 entrevistas semiestructuradas (Bernard, 1994) a habitantes locales. Los entrevistados fueron sugeridos por la casa ejidal, seleccionando en especial a quienes tenían antecedentes de ventas de terrenos y las entrevistas fueron realizadas durante los meses de octubre y diciembre del 2013, a personas con un rango de edad entre los 35 y 70 años, de los cuales $100 \%$ eran hombres que ya que más del $90 \%$ de los dueños del ejido son de este sexo.

\section{Dinámicas de cambio del BTC}

El mapa de vegetación potencial sugiere que el BTC cubría 63.320 ha de la zona de estudio, lo que representaba el 73\% del área total. El BTC-perturbado, el BTC conservado y la agricultura de temporal perdieron más de 50 ha, pero menos de 400 ha durante el periodo, por lo que como resultado tuvieron tasas de cambio negativas y menores al $1 \%$ anual. Las zonas de pastizal, agricultura de riego vegetación secundaria de BTC y bosque de pino, perdieron más de 400 ha de extensión y tuvieron tasas de cambio negativas y superiores al $1 \%$ anual. Entre estas categorías estuvieron las mayores tasas de disminución, que correspondieron al pastizal y la vegetación secundaria de BTC. Cinco categorías extendieron su área: el bosque mesófilo, el suelo desnudo y el bosque de pino-encino ganaron menos de 60 ha y su tasa de cambio fue positiva e inferior al $1 \%$ anual, mientras que el bosque de pino perturbado y las áreas urbanas ganaron más de 1.000 ha y tuvieron las tasas de cambio positivas más elevadas (superior al 1,5\% anual).

La matriz de transición sugiere tres dinámicas de cambio, una en la parte alta de la subcuenca, otra en la zona del BTC y otra en la zona urbana, de pastizales y agrícola. En la parte alta de la subcuenca las transformaciones sugieren procesos naturales de recuperación e impacto humano ya que el bosque 
de pino conservado mostró probabilidades de persistencia superiores al $80 \%$, debido a que en este y su vegetación perturbada ocurrió una recuperación hacia áreas conservadas (4\%). Sin embargo, el $16 \%$ de bosque de pino conservado se convirtió en bosque de pino perturbado, $7 \%$ del bosque de pino perturbado y $1,5 \%$ del bosque de pino conservado se transformaron en pastizales.

La dinámica del BTC y su vegetación secundaria mostró que la deforestación para esta categoría se detuvo, pero sin presentar aumentos de superficie. Más de un $98 \%$ de las áreas de BTC conservado se mantuvieron; sin embargo, las áreas de BTCp y de vegetación secundaria del BTC no se recuperaron hacia bosque conservado sino que se deterioraron, en conjunto hacia pastizales y zonas urbanas. Entre los bosques presentes en la subcuenca, el BTC es el más fragmentado en la medida en que para el año 2012 se detectaron 246 fragmentos en condiciones primarias, 418 perturbados y 711 fragmentos de vegetación secundaria derivada del BTC.

La dinámica en el periodo analizado muestra que las zonas urbanas crecieron, principalmente a costa de pastizales, zonas agrícolas y vegetación secundaria. Los pastizales procedentes del BTC se transformaron a zonas urbanas, mientras que se mantuvieron los pastizales que se originaron desde áreas de bosques templados. Como resultado, el pastizal fue la cobertura que más terreno perdió. Más del $10 \%$ de las zonas de agricultura de riego se transformaron en zona urbana, siendo esta última cobertura la que no perdió superficie y siendo el uso de suelo que más se extendió (>7.000 ha).

En las áreas del BTC, el 78\% de los propietarios de terrenos entrevistados vendieron total o parcialmente sus terrenos. El $42 \%$ de los propietarios no estaba interesado en vender, pero recibieron ofertas de compra que los indujeron a vender; otro $36 \%$ vendió porque sus descendientes ya no trabajan las tierras, ya sea porque estaban estudiando o porque migraron hacia Estados Unidos. El resto de quienes vendieron lo hicieron porque necesitaban dinero. Los terrenos vendidos tenían entre 3 y 12 años de abandono. Una vez vendidos, el $67 \%$ de los terrenos fueron destinados para la ampliación de la zona urbana,
$22 \%$ permanecen como zona agrícola y $11 \%$ siguen abandonados.

A escala regional, la investigación permitió encontrar que en la subcuenca del río Apatlaco hay procesos de recuperación y de deterioro de los ecosistemas terrestres, y que estos procesos se estratifican altitudinalmente. No obstante, la mayor parte de los cambios en área correspondieron al crecimiento de la zona urbana, en detrimento de áreas donde los ecosistemas terrestres no se recuperaron. Dicho patrón suele quedar oculto en estudios de escalas más generales (Miles et al., 2006).

En la parte alta de la subcuenca, la recuperación del bosque de pino fue menor que el cambio de este bosque hacia zonas perturbadas, como se ha señalado en otras reservas (Whitehurst et al., 2009). La ampliación del área perturbada de bosques fríos puede obedecer a la actividad de extracción de madera en la zona. Para la zona del corredor biológico Chichinautzin, un trabajo reciente encontró que la frecuencia de zonas con evidencia de extracción de madera es del $67,7 \%$, siendo parecida a la de los bosques neotropicales con similares características (Cruz-Fernandez et al., 2011). Sin embargo, los datos de este trabajo sugieren que la extracción de madera podría afectar la densidad de árboles, hasta hacerla evidente desde las imágenes que se utilizan para este tipo de estudios y como se ha señalado para el BTC (Whitehurst et al., 2009).

Para la zona de BTC, la hipótesis fue rechazada, pues la tasa anual de cambio que se obtuvo fue de menos de un décimo de pérdida anual, mientras que trabajos previos la reportaron en 1,4\% anual (Trejo \& Dirzo 2000). Esto muestra que en la década 20022012 se desaceleró la deforestación del BTC de la zona de estudio. La diferencia con la estimación para el periodo 1973-1989, puede estar vinculada a las políticas aplicadas en el periodo de 1970 a 1976, cuando se otorgó tanto tierra, como créditos para eliminar la vegetación (Merino-Pérez, 2004). A raíz de estas políticas (1977- 1985), el área dedicada a la ganadería se incrementó en más de 100\% (Banco Mundial, 1995). La desaceleración de la deforestación que se observó, en lugar de ser un resultado de políticas de conservación eficientes, puede ser producto de la 
falta de recursos para la explotación agrícola o de que las áreas de bosque conservado no son aprovechables para el desarrollo de actividades agrícolas.

\section{Conclusiones}

Las evidencias de este trabajo muestran que aunque se frenó la deforestación y disminuyó la cobertura de vegetación secundaria, no hubo un incremento en la cobertura de los ecosistemas primarios. En 1992, en México ocurrió una modificación de la Constitución, que eliminó restricciones para la compraventa de tierras ejidales (Secretaría de Gobernación, 1992). Las entrevistas revelaron que la mayor parte de los territorios de BTC vendidos, estaban abandonados porque los propietarios no tenían dinero para trabajarlos y/o habían migrado a Estados Unidos. La compra-venta de tierras ejidales estuvo relacionada directamente con la conversión de tierras de cultivo activas y/o abandonadas en zonas urbanas.

Estimamos que el BTC cubría más del $70 \%$ de la subcuenca del río Apatlaco y actualmente solo queda menos del $20 \%$, siendo más abundantes los fragmentos de BTC perturbado y la vegetación secundaria derivada de BTC. Los datos muestran que el BTC conservado no se ha recuperado. La recuperación del BTC es baja debido a que la vegetación secundaria y los pastizales derivados de BTC cambian hacia áreas urbanas. Los 246 fragmentos de BTC en condiciones primarias y 418 fragmentos perturbados están crónicamente reducidos y aislados, no aumentaron su área y la matriz que los rodea podría impedir el movimiento de organismos, especialmente la presencia de áreas urbanas.

EI BTC es el bosque más fragmentado de la subcuenca, lo que concuerda con la apreciación del estado de este bosque en la costa del Pacífico mexicano (Miles et al., 2006). Además, el número de fragmentos de BTC conservado duplica a los de bosque de pino, cuadriplica a los del bosque de encino y sextuplican a los de bosque de pino-encino. Su alta fragmentación se revela en que solo hay tres fragmentos de BTC mayores a 100 ha, de ellos dos son mayores a 128 ha y la mayor parte de los fragmentos están entre 2 y
16 ha. En contraste, para el bosque de pino, el bosque de encino y el bosque de pinoencino hay al menos un fragmento de más de 500 ha.

La reducción del BTC en sus diferentes estados de conservación y su alta fragmentación es una amenaza directa a la biodiversidad, pues algunas especies de flora y fauna podrían requerir de áreas de BTC contiguas para sostener sus poblaciones (Henle et al., 2004; Raghubanshi \& Tripathi, 2009). De continuar la reducción y la fragmentación del BTC, aumentaría la amenaza para la supervivencia de muchas especies de plantas y animales. Raghubanshi y Tripathi (2009) encontraron que en el BTC, las comunidades vegetales con una menor área son también las que muestran una menor riqueza y diversidad de especies.

Es importante monitorear constantemente los cambios en las coberturas vegetales en escala regional, para proveer información actualizada, evaluar tendencias y comprender su comportamiento en el transcurso del tiempo (Ernst et al., 2013; Miles et al., 2006 Whitehurst et al., 2009). En la escala regional, el monitoreo permite señalar acciones que corrigen las tendencias de cambio: por ejemplo, a escala global solo se percibe a la densidad poblacional como un factor que afecta al BTC (Miles et al., 2006), pero en la escala regional se detecta que es el crecimiento urbano, el mecanismo que impide la recuperación del BTC. En la subcuenca estudiada, las áreas naturales protegidas no incluyen BTC, y se permite la expansión urbana sobre áreas con vegetación secundaria. Para el BTC de esta región, es necesaria la protección de áreas que sirvan de amortiguamiento a los fragmentos y permitan que el BTC se recupere. Además es prioritario el estudio de los efectos de la reducción y aislamiento crónico del BTC sobre los organismos.

\section{Referencias bibliográficas}

ANDERSON, J.R.; HARDY, E.E.; ROACH, J.T. \& WITMER, R.E. A land use and land Cover classification system for use with remote sensor data. Washington: Geological Survey Professional Paper No. 964, U.S. Government Printing Office, 1976. 
BERNARD, R. Structured interviewing. In: BERNARD, R. (editor). Research methods in cultural anthropology: qualitative and quantitative approaches. London: Altamira Press, 1994, p.237-255.

Ceballos, G. \& GARCíA, A. Conserving neotropical biodiversity: the role of dry forest in western México. Conservation Biology.1995, Vol. 9, № 6, p.1349-1353.

CONTRERAS-MACBEAT, T.; JARAMILLO, F.; MONROY, R. y BOYÁS-DELGADO, J.C. La diversidad biológica en Morelos. Estudio de Estado. México: CONABIO/Universidad Autónoma del Estado de Morelos, 2004.

CRUZ-FERNÁNDEZ, Q.T.; ALQUICIRAARTEAGA M.L. \& FLORES-PALACIOS, A. Is orchid species richness and abundance related to the conservation status of oak forest? Plant Ecology, 2011, Vol. 212, No 7 p.1091-1099.

ERNST,C.; MAYAUX, P.;VERHEGGHEN, A.; BODART, C.; CHRISTOPHE, M. \& DEFAURNY, P. National forest cover change in Congo Basin: Deforestation, reforestation, degradation and regeneration for years 1990, 2000 and 2005. Global Change Biology, 2013, Vol. 19, No 4 p. 1173-1187.

FLORES, O. y GEREZ, P. Conservación en México: Síntesis sobre vertebrados terrestres, vegetación y uso de suelo. México: Instituto Nacional de Investigaciones sobre recursos bióticos/Conservación internacional, 1994.

FUEYO, L. Manglares de México. México: Comisión Nacional para el Conocimiento y uso de la Biodiversidad, 2008.

GOETZ, S.J.; BACCINI, A.; LAPORTE, N.T.; JOHNS, T.; WALKER, W.; KELLNDORFER, J.; HOUGHTON, R.A. \& SUN, M. Mapping and monitoring carbon stocks with sateIlite observations: a comparison of methods. Carbon Balance and Monitoring, 2009, Vol. 4, $\mathrm{N}^{\circ} 2$, p. 1-7.

HE, F. \& HUBBELL, P. Species-area relationships always overestimate extinction rates from habitat loss. Nature, 2011, Vol. 473, p. 368-371.
HENLE, K.; DAVIES, K.F.; KLEYER, M.; MARGULES, C. \& SETTELE, J. Predictors of species sensitivity to fragmentation. Biodiversity and Conservation, 2004, Vol.13, № 1, p. 207-251.

MAS, J.F.; SORANI, V. y ÁLVAREZ, R. Elaboración de un modelo de simulación del proceso de deforestación. Investigaciones Geográficas, 1996, № 5, p. 43-47.

MERINO-PÉREZ, L .Conservación o deterioro. El impacto de las políticas en las instituciones comunitarias y en las prácticas de usos de los recursos forestales en México. México: Instituto Nacional de Ecología, 2004.

MILES, L.; NEWTON, A.C.; DEFRIES, R.S.;RAVILIOUS, C.; MAY, I.; BLYTH, S.; KAPOS, V. \& GORDON, J.E. A global overview of the conservation status of tropical dry forest. Journal of Biogeogaphy, 2006, Vol. 33, p. 491-505.

ORGANIZACIÓN DE LAS NACIONES UNIDAS PARA LA ALIMENTACIÓN Y LA AGRICULTURA (FAO). Evaluación de los recursos forestales mundiales 2005 - hacia la ordenación forestal sostenible. Roma: Estudio FAO, Montes $N^{\circ}$ 47, 2006.

PALACIOS-PRIETO, J.L.; BOCCO, G.; VELÁZQUEZ, A.; MAS. J.F, TAKAKI-TAKAKI, F.; VICTORIA, A.; LUNA-GONZÁLEZ, L.; GÓMEZ-RODRÍGUEZ, G.; LÓPEZ-GARCÍA, J.; PALMA-MUÑOZ, M.; TREJO-VÁZQUEZ, I.; PERALTA HIGUERA, A.; PRADO-MOLINA, J.; RODRÍGUEZ- AGUILAR, A.; MAYORGASAUCEDO, R. y GONZÁLEZ-MEDRANO, F. La condición actual de los recursos forestales en México: resultados del Inventario Forestal Nacional. Investigaciones Geográficas, 2000, No 43 p. $183-203$.

SAGARPA. Programa Nacional de Microcuencas. México: Secretaría de Agricultura, Ganadería, Desarrollo rural, Pesca y Alimentación, 2006.

SECRETARIA DE GOBERNACIÓN. Decreto por el que se Reforma el artículo 27 de la Constitución política de los Estados Unidos Mexicanos. Diario Oficial de la Federación, Vol. CDLX, 1992, N³ ${ }^{\circ}$, enero 6. 
SEMARNAT. Manual del Proceso de Ordenamiento Ecológico. Anexo 5. Análisis de aptitud con técnicas multicriterio. México: Secretaría de Medio Ambiente, Recursos Naturales y Pesca, 2012.

RAGHUBANSHI, A.S. \& TRIPATHI, A. Effect of disturbance, habitat fragmentation and alien invasive plants on floral diversity in dry tropical forests of Vindhyan highland: a review. Tropical Ecology, 2009, Vol. 50, № 1 , p. 57-69.

RINCÓN, E. \& HUANTE, P. Growth responses of tropical deciduous tree seedlings to contrasting light conditions. Trees, 1993, N ${ }^{\circ}$ 7, p. 202-207.

RZEDOWSKI, J. Diversidad y orígenes de la flora fanerogámica de México. Acta Botánica Mexicana, 1991a, No 14, p. 3-21.
RZEDOWSKI, J. El endemismo en la flora fanerogámica mexicana: una apreciación analítica preliminar. Acta Botánica Mexicana, 1991b, No 15, p. 47-64.

TREJO, I. \& DIRZO, R. Deforestation of seasonally dry tropical forests: a national and local analysis in Mexico. Biological Conservation, 2000, Vol. 94, № 2 p. 133-142.

WHITEHURST, A.S.; SEXTON, J.O. \& DOLLAR, L. Land cover change in western Madagascar's dry deciduous forests: A comparison of forest changes in and around Kirindy Mite National Park. Oryx, 2009, Vol 43, No 2, p. 275-283.

WORLD BANK Estudio de revisión del sector forestal y conservación de recursos. Report 13114-ME. México: Oficina Regional de América Latina y el Caribe, 1995. 
